\title{
Structural and Biochemical Studies on the Reaction Mechanism of Uridine- Cytidine Kinase
}

Fumiaki Tomoike, Noriko Nakagawa, Seiki Kuramitsu, Ryoji Masui

\begin{tabular}{|c|l|}
\hline Citation & The Protein Journal, 34(6); 411-420 \\
\hline Issue Date & $2015-12$ \\
\hline Type & Journal article \\
\hline Text version & Author \\
\hline $\begin{array}{c}\text { Supplementa } \\
\text { ry material }\end{array}$ & Supplementary material is available at $\underline{\text { https://doi.org/10.1007/s10930-015-9636-8 }}$ \\
\hline Rights & $\begin{array}{l}\text { This is a post-peer-review, pre-copyedit version of an article published in The Protein } \\
\text { Journal. The final authenticated version is available online at: } \\
\text { https://doi.org/10.1007/s10930-015-9636-8 }\end{array}$ \\
\hline DOI & $10.1007 / \mathrm{s} 10930-015-9636-8$ \\
\hline
\end{tabular}

\author{
Self-Archiving by Author(s) \\ Placed on: Osaka City University
}


Structural and biochemical studies on the reaction mechanism of uridine-cytidine kinase

Fumiaki Tomoike $^{1,2}$, Noriko Nakagawa ${ }^{3}$, Seiki Kuramitsu ${ }^{1,3}$, and Ryoji Masui ${ }^{3,4}$

1 Graduate School of Frontier Biosciences, Osaka University, 1-3 Yamadaoka, Suita, Osaka 565-0871, Japan

2 Present address: Institute of Industrial Science, The University of Tokyo, 4-6-1 Komaba, Meguro-ku, Tokyo 153-8505, Japan

3 Department of Biological Sciences, Graduate School of Science, Osaka University, 1-1 Machikaneyama-cho, Toyonaka, Osaka 560-0043, Japan

4 Division of Biology \& Geosciences, Graduate School of Science, Osaka City University, 3-3-138 Sugimoto, Sumiyoshi-ku, Osaka 558-8585, Japan

\section{Correspondence}

R Masui, Division of Biology \& Geosciences, Graduate School of Science, Osaka City University, 3-3-138 Sugimoto, Sumiyoshi-ku, Osaka 558-8585, Japan. Telephone: +81-6-6605-2819. E-mail: rmasui@sci.osaka-cu.ac.jp 


\begin{abstract}
Uridine-cytidine kinase catalyzes phosphorylation of the pyrimidine nucleosides uridine and cytidine and plays an important role in nucleotide metabolism. However, the detailed molecular mechanism of these reactions remains to be elucidated. Here, we determined the structure of the ternary complex of uridine-cytidine kinase from Thermus thermophilus HB8 with both cytidine and $\beta, \gamma$-methyleneadenosine 5'-triphosphate, a non-hydrolysable ATP analogue. Substrate binding is accompanied by substantial domain movement that allows the substrate-binding cleft to close. The terminal phosphodiester bond of the ATP analogue is in an ideal location for an inline attack of the 5'-hydroxyl group of cytidine. Asp40 is located near the 5'-hydroxyl group of cytidine. Mutation of this conserved residue to Asn or Ala resulted in a complete loss of enzyme activity, which is consistent with the notion that Asp40 acts as a general base that activates the 5'-hydroxyl group of cytidine. The $\mathrm{pH}$ profile of the activity showed an apparent $\mathrm{p} K_{\mathrm{a}}$ value of 7.4. Based on this structure, a likely mechanism of the catalytic step is discussed.
\end{abstract}

\title{
Keywords
}

uridine-cytidine kinase; phosphate transfer; crystal structure; mutational study; pyrimidine nucleoside; $\mathrm{pH}$ dependence

\begin{abstract}
Abbreviations
AMPPCP, $\beta, \gamma$-methyleneadenosine 5'-triphosphate; CMP, cytidine monophosphate; cv, column volume; NMP, nucleoside monophosphate; $t \mathrm{tUCK}$, uridine-cytidine kinase in T. thermophilus HB8; UCK, uridine-cytidine kinase; WT, wild type
\end{abstract}




\section{Introduction}

Uridine-cytidine kinase (UCK) [EC number: 2.7.1.48] is an important enzyme in pyrimidine nucleotide biosynthesis $[1,2]$. UCK catalyzes the transfer of phosphate from a phosphate donor to a phosphate acceptor. In general, substrates of UCK are cytidine or uridine as phosphate acceptor and any nucleoside triphosphate except for cytidine triphosphate and uridine triphosphate as phosphate donor. UCK is the rate-limiting enzyme in the salvage pathway of pyrimidine nucleotides [3]. Moreover, UCK is known to be overexpressed in some cancer cells $[4,5]$. Recent genomic analyses revealed that most organisms possess UCK family proteins. Interestingly, UCK has been reported to dominate the activation of some nucleoside anticancer drugs and of the two human UCKs, UCK2 plays a crucial role in activating anti-tumor prodrugs in cancer cells $[4,6]$.

It is now more than five decades since the discovery of UCK in mammalian cells [7, 8]. Given the importance of UCK in the medical and health-related fields, it is perhaps surprising that the structure-function relationship of this enzyme is still not fully understood. Kinetic analysis of UCK family proteins indicated that the reaction proceeds by a sequential mechanism and a ternary complex with both substrates is formed as the first step of the reaction [9]. The overall structure of UCK belongs to the nucleoside monophosphate (NMP) kinase fold family [10]. Ligand-free, nucleoside-bound and product-bound structures of UCK were determined $[11,12]$. These studies revealed that both substrates bind to the cleft in the UCK structures. However, the structure of a ternary complex of UCK with both substrates (i.e., phosphate donor and acceptor) has not been determined. A lack of knowledge concerning the structure of the ternary complex with both substrates present hinders a complete understanding of the molecular mechanism of the phosphate transfer from ATP to nucleoside. Although a model of the initial stage of the UCK catalyzed reaction has been proposed $[11,13]$, there has been no experimental evidence to support this mechanism.

Here, we report the ternary structure of UCK from Thermus thermophilus HB8 (ttUCK) with both substrates. Tomoike et al. previously reported the crystal structures of the ligand-free form and binary complex of ttUCK with cytidine monophosphate (CMP), a product [12]. Although ttUCK has 
cytidine-selective kinase activity [12], it displays 33\% sequence identity with human UCK2 and the structure of the active site was the same as that of human UCK2 [11]. The results described in this paper provide some insight into the mechanism of the reaction catalyzed by UCK.

\section{Materials and Methods}

\subsection{Overexpression and Purification of ttUCK}

Overexpression of ttUCK was performed as described previously [12]. The cells were lysed by sonication in $50 \mathrm{mM}$ Tris- $\mathrm{HCl}, 100 \mathrm{mM} \mathrm{KCl}$ and $2 \mathrm{mM}$ ethylenediaminetetraacetic acid, $\mathrm{pH} 8.0$, and the lysate was incubated at $70{ }^{\circ} \mathrm{C}$ for $1 \mathrm{~h}$. After centrifugation, ttUCK was precipitated with ammonium sulfate and redissolved in $20 \mathrm{mM}$ Tris- $\mathrm{HCl}$ and $1.0 \mathrm{M}$ ammonium sulfate, $\mathrm{pH} 8.0$, and the solution was loaded onto a Toyopearl Phenyl-650M column (Tosoh) equilibrated with $20 \mathrm{mM}$ Tris- $\mathrm{HCl}$ and $1.0 \mathrm{M}$ ammonium sulfate, $\mathrm{pH}$ 8.0. After washing with $0.5 \mathrm{M}$ ammonium sulfate, bound proteins were eluted with a linear gradient of $0.5-0.2 \mathrm{M}$ ammonium sulfate. The fractions containing the target protein were dialyzed and loaded onto a Toyopearl SP-650 column (Tosoh) equilibrated with $20 \mathrm{mM}$ potassium phosphate, $\mathrm{pH}$ 6.2. Bound proteins were eluted with a linear gradient of $0-0.2 \mathrm{M} \mathrm{KCl}$ in $20 \mathrm{mM}$ potassium phosphate, $\mathrm{pH}$ 6.2. The fractions containing the target protein were pooled and concentrated using a Vivaspin concentrator. The concentrated protein solution was then subjected to size-exclusion chromatography using a Superdex 200 10/300 GL column (GE Healthcare Biosciences, Piscataway, $\mathrm{NJ})$ equilibrated with $50 \mathrm{mM}$ Tris- $\mathrm{HCl}$ and $100 \mathrm{mM} \mathrm{KCl}, \mathrm{pH}$ 8.0. Size-exclusion chromatography was carried out on an ÄKTA explorer system (GE Healthcare Biosciences). At each chromatography step, the purity of the various fractions was assessed by sodium dodecyl sulfate polyacrylamide gel electrophoresis. The concentrations of the purified proteins were determined by using molar absorption coefficients, calculated to be $11,200 \mathrm{M}^{-1} \mathrm{~cm}^{-1}$ at $278 \mathrm{~nm}$ for ttUCK [14].

\subsection{Crystallization and Structural Determination}

To obtain a crystal of a ternary complex of ttUCK, we tried to crystallize ttUCK in the presence of 
cytidine and adenosine $\beta, \gamma$-imidoadenosine 5 '-triphosphate or $\beta, \gamma$-methyleneadenosine 5 '-triphosphate (AMPPCP) using Crystal Screen 1 and Crystal Screen 2 from Hampton Research (Aliso Viejo, CA), and Precipitant Synergy, Cryo I and Cryo II Screen from Emerald Biosystems (Bainbridge Island, Washington, USA) by the 96-well sitting-drop vapor-diffusion method at $20^{\circ} \mathrm{C}$ [15]. We obtained crystals under several conditions of these crystallization trials and collected the diffraction data for structure determination. However, most of them did not contain an ATP analogue judging from the electron density map. As a result, we found that No. 46 condition from Crystal Screen 2 yielded diffraction-quality crystals of the ttUCK-cytidine-AMPPCP complex.

The protein solution for crystallization contained $5.6 \mathrm{mg} / \mathrm{mL}$ ttUCK and $1 \mathrm{mM} \mathrm{MgCl}, 1 \mathrm{mM}$ nucleoside and $1 \mathrm{mM}$ AMPPCP. A $1 \mu \mathrm{L}$ aliquot of each protein solution was mixed with an equal volume of a precipitant solution. Diffraction-quality crystals were obtained in a solution of $50 \%$ of No $46(0.1 \mathrm{M} \mathrm{NaCl}, 0.1 \mathrm{M}$ bicine, $\mathrm{pH} 9.0$, and 20\% (v/v) polyethylene glycol monomethyl ether 550$)$ from Crystal Screen 2 at $20^{\circ} \mathrm{C}$. Data were collected with the RIKEN Structural Genomics Beamline II (BL26B2) [16] at SPring-8 (Hyogo, Japan) and processed using the HKL-2000 program suite [17]. Structures were solved by using a molecular replacement method with Molrep [18]. The coordinates of the ttUCK-CMP complex structure (PDB code 3ASZ) were used as the starting model. Model refinement was carried out by using the programs Xtalview [19] and CNS [20]. Evaluation of the ttUCK structure was performed by PROCHECK [21]. The molecular models of cytidine and AMPPCP were obtained from website HIC-Up [22]. The model was analyzed by Chimera [23] and PyMoL (http://pymol.sourceforge.net/). The final model was deposited into the Protein Data Bank (PDB code 3W34).

\subsection{Site-directed Mutagenesis}

Site-directed mutagenesis of ttUCK was performed by using the method of Iwai et al. [24] with some modifications. Overexpression and purification of the mutant proteins were performed in a manner similar to that used for the wild-type (WT) enzyme. 


\subsection{Enzyme Assay}

Reaction solutions contained $50 \mathrm{mM}$ 4-(2-hydroxyethyl)-1-piperazineethanesulfonic acid, $100 \mathrm{mM}$ $\mathrm{KCl}, \mathrm{pH} 7.3,1 \mathrm{mM} \mathrm{MgCl} 2,1 \mathrm{mM}$ cytidine, $500 \mu \mathrm{M}$ ATP and $142 \mathrm{nM}$ ttUCK WT or a mutated form of the enzyme (D40A, D40N or H41A). Reactions were performed at $25^{\circ} \mathrm{C}$ and stopped by mixing with a half volume of $100 \mathrm{mM}$ ethylenediaminetetraacetic acid, $\mathrm{pH}$ 8.0. After filtration through a $0.22 \mu \mathrm{m}$ filter unit, the reaction products were applied onto a MonoQ HR 5/5 column (GE Healthcare Biosciences) equilibrated in buffer A (20 mM Tris-HCl, pH 8.0) using an ÄKTA system. Bound material was eluted using a gradient from buffer A to $100 \%$ buffer B $(20 \mathrm{mM}$ Tris- $\mathrm{HCl}$ and $500 \mathrm{mM}$ $\mathrm{NaCl}, \mathrm{pH} 8.0$ ) as follows: 2 column volumes (cv) of buffer $\mathrm{A} ; 3 \mathrm{cv}$ of a linear gradient $20-60 \%$ buffer $\mathrm{B}$; and $1.5 \mathrm{cv}$ of $100 \%$ buffer B. Enzyme activity was determined from the ratio of peak areas of substrate to product.

We assumed that there is one residue that is related to catalysis by ttUCK (Scheme 1). In this scheme, its ionizable side chain must be deprotonated to produce the only one active species of the enzyme $\left(E^{-}\right)$.

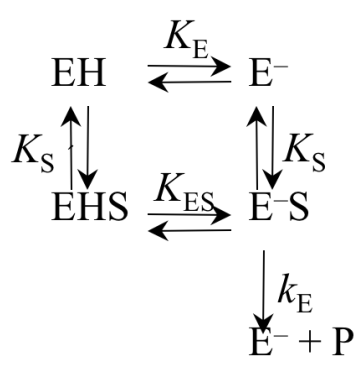

Scheme 1

Assuming this scheme, the following equations could be generated [25].

$$
\begin{aligned}
K_{\mathrm{E}} & =\left[\mathrm{E}^{-}\right]\left[\mathrm{H}^{+}\right] /[\mathrm{EH}] \\
K_{\mathrm{ES}} & =\left[\mathrm{E}^{-} \mathrm{S}\right]\left[\mathrm{H}^{+}\right] /[\mathrm{EHS}] \\
K_{\mathrm{S}} & =\left[\mathrm{E}^{-}\right][\mathrm{S}] /\left[\mathrm{E}^{-\mathrm{S}}\right] \\
K_{\mathrm{S}^{\prime}} & =[\mathrm{EH}][\mathrm{S}] /[\mathrm{EHS}]
\end{aligned}
$$




$$
[\mathrm{E}]_{0}=\left[\mathrm{E}^{-}\right]+[\mathrm{EH}]+\left[\mathrm{E}^{-} \mathrm{S}\right]+[\mathrm{EHS}]
$$

When the concentration of cytidine were sufficient for maximal velocity ([S] $\left.>>\mathrm{K}_{\mathrm{S}}\right), k_{\text {cat }}$ values under various $\mathrm{pH}$ conditions were determined using the following equation:

$$
k_{\text {cat }}=k_{\mathrm{E}} /\left(1+\left[\mathrm{H}^{+}\right] / K_{\mathrm{ES}}\right)
$$

The value of $\mathrm{p} K_{\mathrm{a}}$, which is $\mathrm{p} K_{\mathrm{ES}}$, was calculated by fitting the data to Equation 6 using the software Igor 4.03 (WaveMetrics, Portland, OR).

\section{Results}

\subsection{Overall Structure of ttUCK}

To obtain a crystal of a ternary complex of ttUCK containing a non-hydrolysable ATP analogue, we tried to crystallize ttUCK in the presence of cytidine and $\beta, \gamma$-imidoadenosine 5 '-triphosphate or AMPPCP (see details in Materials and Methods). Although diffraction-quality crystals were produced under several conditions, a crystal of a ternary complex was obtained under one condition, which contained AMPPCP. The crystal structure of ttUCK in complex with cytidine and AMPPCP was determined at $1.9 \AA$ resolution (Table 1 and Fig. 1). The structure is referred to as a ternary complex in this report. There are two ttUCK molecules in an asymmetric unit (Fig. 1a). Previous studies using size-exclusion chromatography had suggested that ttUCK forms a dimer in solution [12]. Comparison between the two subunits in the asymmetric unit indicated a small root-mean-square deviation value $(0.3 \AA)$ of the main chain atoms. Although a few amino acid residues at both the $\mathrm{N}$ - and $\mathrm{C}$-termini of the subunit B could not be determined, the overall structure was successfully solved for both subunits. The structure of a subunit is composed of ten $\alpha$-helices and eight $\beta$-strands and divided into four domains: the core domain, the LID domain, the NMP-binding domain, and the $\beta$ hairpin domain (Fig. 1b). The domains are named according to the nomenclature used for human UCK2 structure [11]. The core domain is composed of five $\beta$-strands, sandwiched by seven $\alpha$-helices, and contains a dimerization interface (Fig. 1a). The LID domain is composed of two $\alpha$-helices, one of which is extended towards the core domain. The $\beta$ hairpin domain is composed of three $\beta$-strands. The conformation of the $\beta$ hairpin 
domain is slightly different in the two subunits, suggesting this domain possesses a degree of flexibility. The NMP-binding domain is composed of one loop and two short $\alpha$-helices. A long cleft is formed between the LID domain and the other domains. The positions of the amino acid residues that form the cleft are identical between subunits. Half of this cleft is positively charged.

\subsection{Ligands in the Ternary Complex.}

The electron density of ligand molecules show they are located in the long cleft (Fig. 2). The electron density of cytidine was clearly identified (Fig. 2a). However, despite the high resolution obtained for the overall structure, the quality of the electron density of AMPPCP was surprisingly poor (Fig. 2b). The shape of the electron density contours indicated a characteristic tetrahedral density, in which the $\gamma$-phosphate group of AMPPCP could be easily modeled. In the electron density contours of the adjacent $\alpha$ and $\beta$ phosphate groups, non-bridging oxygen atoms were difficult to model. The nucleoside moiety of AMPPCP could not be detected in the density map. Consequently, our discussion concerning the bound ATP (analogue) is limited to its phosphate groups. In addition, the electron density of $\mathrm{Mg}^{2+}$ could not be found in the structure of ttUCK despite the presence of $\mathrm{MgCl}_{2}$ in the crystallization solution.

Cytidine binds to ttUCK via several interactions. As shown in Fig. 3a, the 2'- and 3'-hydroxyl groups of the sugar moiety interact with Asp60 and Arg142. The O2 and N3 atoms of the base moiety form two hydrogen bonds with $\operatorname{Arg} 152$. The $\mathrm{O} 2$ atom also hydrogen bonds with Gln160. The three residues Arg142, Arg152 and Gln160 are from the LID domain. In addition, hydrophobic residues, Tyr43, Tyr59, Tyr88, Phe90 and Tyr93, which are from the NMP-binding and $\beta$ hairpin domains, form a hydrophobic pocket. This pocket might greatly contribute to binding of the base. Structural analyses of GMP kinase and AMP kinase have shown that the NMP-binding domain is the only domain that interacts with the phosphate donor $[4,26,27]$. Therefore, the contribution of the LID and $\beta$ hairpin domains to the interaction with cytidine could be considered a characteristic of UCK among NMP kinase fold family members. 
Thr15, Ala16, Gly18, Lys19, Thr20 and Arg145 are located around the phosphate moiety of AMPPCP (Fig. 3a). The side chains of basic residues and main chains of hydrophobic residues form a narrow positively-charged pocket that might promote ATP binding.

This is the first report of the structure of a ternary complex of UCK with both phosphate donor (ATP analogue) and acceptor (a nucleoside) in the physiological direction of UMP formation. The overall structure of the ternary complex was compared with those of the ligand-free form and CMP-bound form (a binary complex) reported previously [12]. When the ternary complex is compared with the ligand-free form, the main-chain rmsd was not particularly large $(0.8 \AA)$. Upon substrate binding, the LID and $\beta$ hairpin domains move towards the cleft by up to $5.2 \AA$ (at the $\alpha 8-\alpha 9$ loop) and $6.1 \AA$ (at the $\beta 3-\beta 4$ loop), respectively, relative to the core domain (Fig. 4a). However, structural comparison of the ternary complex with the binary complex showed the main chain rmsd was only 0.3 $\AA$ (Fig. 4b). This high degree of similarity is consistent with the identical space group of the crystals of these complexes.

\subsection{Catalytic Mechanism of Phosphate Transfer}

Comparison between the positions of the substrate and the product has facilitated detailed insight into the reaction mechanism of UCK (Fig. 5). Specifically, the positions of the base moiety of cytidine and CMP are almost the same, whereas the position of the $\mathrm{C}^{\prime}$ in the ribose moiety is slightly altered between substrate and product (described later). In the ternary complex, the distance between the 5 '-oxygen atom of cytidine and the $\gamma$-phosphorus atom of AMPPCP is $3.3 \AA$. This distance is too far to allow these ligands to engage in direct transfer of the phosphate group. It should also be emphasized that this study used AMPPCP, a non-hydrolysable ATP analogue. The $\beta, \gamma$-phosphate bridge oxygen atom (in this case, the carbon atom) and the $\gamma$-phosphorus atom of ATP (AMPPCP), and the 5'-oxygen atom of cytidine are positioned along a line (Fig. 5).

The prerequisite for a nucleophilic attack of the 5'-hydroxyl group on the phosphodiester is its deprotonation by a general base. Asp 40 is the only acidic residue located near the 5 '-hydroxyl group of 
cytidine (Fig. 5). This residue is completely conserved among all UCKs (Fig. 3b and Online Resource 1), indicating the importance of Asp40 for the reaction. To verify the contribution of Asp40 to the catalysis of ttUCK, we used site-directed mutagenesis to replace this Asp residue with either Asn (D40N) or Ala (D40A). Phosphorylation reaction of cytidine by WT and mutant ttUCKs was performed at $25{ }^{\circ} \mathrm{C}$, and the reaction products were analyzed at several time points by high-performance liquid chromatography (Fig. 6a). WT ttUCK converted most of the cytidine to CMP within $1 \mathrm{~h}$, whereas D40N and D40A mutant proteins gave no detectable reaction even after $3 \mathrm{~h}$ (Fig. 6b). This result indicated that Asp40 of ttUCK is indispensable for the enzyme activity. These findings suggest that Asp40 acts as a general base to deprotonate the hydroxyl group. The side chain of Asp40 might be closer to the 5'-hydroxyl group at the catalytic step than at substrate-binding step.

Appropriate positioning of the phosphodiester group is another prerequisite for an inline attack of the 5'-hydroxyl group of cytidine. Such a spatial arrangement would facilitate formation of a hypothetical pentavalent transition state. The $\beta, \gamma$-phosphate bridge oxygen atom and the $\gamma$-phosphorus atom of ATP (in this case, AMPPCP), and the 5'-oxygen atom of cytidine are positioned along a line (Fig. 5). This observation supports the idea of direct phosphoryl transfer without formation of a phosphoenzyme intermediate.

In the ternary complex, the side chain of Arg 145 is hydrogen-bonded to a non-bridging oxygen of the $\gamma$-phosphate of AMPPCP at a distance of 2.8-3.0 $\AA$ (Fig. 3a and 5). In the CMP-bound structure, the side chains of Arg145 and Arg150 form three hydrogen bonds with the non-bridging oxygen atom of the phosphate group of CMP at a distance of 2.7-2.9 $\AA$ (Fig. 5). These findings suggest involvement of Arg145 (and Arg150) in the catalytic step via interaction with the transferred phosphoryl group. These arginine residues might contribute to stabilization of the transition state because they interact with the terminal phosphate groups of both ligands. Interestingly, the angle of the sugar moiety of cytidine in the ternary complex and CMP in CMP-bound ttUCK differed by $19^{\circ}$. In the binary complex, the distance between these residues and the 5'-oxygen atom of CMP is 3.4-3.9 $\AA$, whereas in the ternary complex the distance between these residues and the 5'-oxygen atom of cytidine is more than $4.8 \AA$. Therefore, the 
interaction with $\operatorname{Arg} 145$ and $\operatorname{Arg} 150$ might cause a conformational change of the ribose in CMP. Comparison of the structures between cytidine-bound and CMP-bound forms of human UCK2 showed no significant differences in the conformation between cytidine and CMP [11]. The distance between the phosphate of CMP and Arg174 of human UCK2, corresponding to Arg150 of ttUCK, was longer (5.0 ̊) than that in ttUCK.

\section{5 pH Dependence of the Activity}

In order to explore the importance of Asp40 for the enzyme activity, we analyzed the dependence of ttUCK activity on $\mathrm{pH}$ across a range of $\mathrm{pH}$ conditions ( $\mathrm{pH} 5$ to 9). A proposed role of Asp40 is the removal of a hydrogen atom from the 5'-hydroxyl group of cytidine, which presumably require deprotonation of the corresponding side chain. If this is the case, ttUCK would be inactivated under acidic conditions. As shown in Fig. 7, the activity of WT ttUCK was high under basic conditions, but drastically reduced between $\mathrm{pH} 8$ to 6 . Below pH 6, WT ttUCK was almost inactivated. The loss of activity under acidic conditions suggested deprotonation of some functional group was indispensable for the enzyme activity. However, theoretical analysis of the $\mathrm{pH}$ profile indicated that the corresponding functional group has an apparent $\mathrm{p} K_{\mathrm{a}}$ value of $7.41 \pm 0.07$. This $\mathrm{p} K_{\mathrm{a}}$ value is more like that of a side-chain imidazole group of His, rather than a side-chain carboxyl group of Asp in water [28].

Except for His41, all other His residues in ttUCK are located far from the active site (Online Resource 2). Asp40 and His41 are adjacently positioned at a distance of $4 \AA$ (Fig. 5). To verify whether His41 could account for the observed $\mathrm{pH}$ dependence of the enzyme-catalyzed reaction, His41 was replaced with Ala and the activity of this mutant (H41A) was measured at various $\mathrm{pH}$ values. H41A showed a similar $\mathrm{pH}$ profile to that of the WT enzyme, although the $\mathrm{p} K_{\mathrm{a}}(7.04 \pm 0.02)$ was slightly different (Fig. 7). In addition, the $k_{\text {cat }}$ value of $8.1 \pm 0.1 \mathrm{~s}^{-1}$ under basic (or alkaline) conditions was also similar to that of the WT enzyme $\left(9.0 \pm 0.2 \mathrm{~s}^{-1}\right)$. These findings indicate that His41 is not significantly involved in the enzyme activity, and thus rules out the possibility that His41 is the functional group with an apparent $\mathrm{p} K_{\mathrm{a}}$ value of 7.4 


\section{Discussion}

This is the first report of the structure of a ternary complex of UCK with both phosphate donor (ATP analogue) and acceptor (a nucleoside). Suzuki et al. previously reported the structure of human UCK2 in complex with CMP and ADP [11], which corresponds to an enzyme-product complex. In our structure, only the phosphate moiety of AMPPCP was included. In contrast, the electron density of ADP in the CMP-ADP complex of human UCK2 was clearly identified [11]. It should be noted, however, that the nucleoside moiety of ADP forms only one hydrogen bond with human UCK2 [11]. In principle, electron density is absent or disordered from regions that are highly flexible. Therefore, our observation implied significant mobility of the nucleoside moiety of bound AMPPCP.

Notably, there was no electron density in the equivalent position to $\mathrm{Mg}^{2+}$ in the structure of human UCK2 [11]. Generally, a divalent metal ion-nucleotide complex like Mg-ATP is the true substrate for enzymes like kinases [29]. Because the overall interaction of AMPPCP with $\mathrm{Mg}^{2+}$ can be considered to be similar to that of ATP, it might be expected that $\mathrm{Mg}^{2+}$ bound to ttUCK under this condition. However, there is also a report that the binding of AMPPCP to $\mathrm{Ca}^{2+}$-ATPase is perturbed in the presence of $\mathrm{Mg}^{2+}$ [30]. This implies that the presence of $\mathrm{Mg}^{2+}$ might perturb the binding of AMPPCP to ttUCK, leading to the apparent lack of $\mathrm{Mg}^{2+}$ in the active site.

The conformation of the ternary complex of ttUCK was essentially similar to that of the CMP-bound form. Our findings suggest that binding of a nucleoside alone to the cleft is sufficient for a large induced fit in UCK. Also, binding of cytidine alone to human UCK2 is known to induce a large conformational change similar to that seen for the CMP-ADP (product) complex [11]. It seems unlikely that the ternary structure determined in this study reflects a conformation of the transition state of UCK. The distance, $3.3 \AA$, between the 5'-oxygen atom of cytidine and the $\gamma$-phosphorus atom of AMPPCP is larger than that for the transition state in the phosphotransfer reaction of ribozymes [31]. AMPPCP cannot be hydrolyzed by this enzyme. Moreover, the structure lacks a divalent cation, which is necessary for the activity of UCK. In the human UCK2 structure, a $\mathrm{Mg}^{2+}$ cation is coordinated to two 
non-bridging oxygen atoms of CMP and ADP [11]. It is probable that $\mathrm{Mg}^{2+}$ partially neutralizes the negative charges of the phosphate groups and helps stabilize the transition state during the $\gamma$-phosphate transfer from ATP to nucleoside.

Asp40 was thought to be a candidate for a general base that activates the 5'-hydroxyl group of cytidine [11]. The results of the mutational study of Asp40 supports the important role of Asp40 in catalysis (Fig. 6). Asp62 in human UCK2, corresponding to Asp40 of ttUCK, is thought to be an important residue for the reaction mechanism [11], but this had not been verified experimentally. In human UCK2-cytidine complex, the side chain of Asp62 is hydrogen-bonded to the 5'-hydroxyl group of cytidine: the distance between Asp62 and cytidine is $2.6 \AA$ (Fig. 8). In contrast, the distance between the side-chain carboxyl group of Asp40 and the 5'-hydroxyl group of cytidine is too far (4.1 $\AA$ ) in the ternary complex. This difference arises from the change in direction of the side-chain carboxyl group between these Asp residues: specially the carboxyl group of Asp40 in ttUCK is not directed towards cytidine. Instead, a water molecule is situated near the 5'-hydroxyl group of cytidine at a distance of 2.6 $\AA$ and close to the terminal phosphate of AMPPCP (2.8 $\AA)$. However, the side chain of Asp40 in the structure of the binary complex projects in the same direction as in that of the ternary complex, despite the absence of a water molecule at the corresponding position. During the catalytic step, the side chain of Asp40 of ttUCK might be directed to cytidine in a similar way to Asp62 of human UCK2.

It remains to be determined which functional group displays an apparent $\mathrm{p} K_{\mathrm{a}}$ of 7.4. Initially, His41 was considered to be a likely candidate for the functional group with the apparent $\mathrm{p} K_{\mathrm{a}}$ value, but this possibility was ruled out by the mutational study (Fig. 7). Alternatively, the apparent $\mathrm{p} K_{\mathrm{a}}$ value of the reaction might reflect a $\mathrm{p} K_{\mathrm{a}}$ of the phosphate group of $\mathrm{ATP}$. The $\mathrm{p} K_{\mathrm{a}}$ values of $\mathrm{ATP}^{3-}$ and $\mathrm{Mg}-\mathrm{ATP}^{-}$are 6.95 and 5.21, respectively [32]. The 5'-hydroxyl group is located only 3.0-3.1 $\AA$ away from the non-bridging oxygen atoms of the $\gamma$-phosphate of AMPPCP. The side chains of Lys19 and Arg145 also interact with non-bridging oxygen atoms at a distance of 2.3-3.0 $\AA$. It has been proposed for other enzymes that the $\gamma$-phosphate oxygen atoms serve as a general base catalyst to induce a phosphoryl transfer mechanism [33]. However, this hypothesis seems inconsistent with the results of the mutational 
study of Asp40.

We thus examined the possibility that the $\mathrm{pH}$-rate profile of $\mathrm{ttUCK}$ activity reflected the $\mathrm{p} K_{\mathrm{a}}$ value of Asp40. The $\mathrm{p} K_{\mathrm{a}}$ values of internal Asp residues have been reported to shift by as much as $5 \mathrm{p} K_{\mathrm{a}}$ units [34] due to the microenvironment around the ionizable group. Specifically, a hydrophobic environment destabilizes the deprotonated form, which elevates the $\mathrm{p} K_{\mathrm{a}}$ of the carboxylic group of Asp residues. As such, the neutral state of the carboxylic groups of buried Asp residues is preferred over the charged one. Our structure of ttUCK shows that Asp40 is not buried in the interior of the protein but faces into a cleft that comprises the active site of the enzyme. Nonetheless, several hydrophobic residues are located near the side chain of Asp40 (Fig. 8a). A similar arrangement is found for Asp62 in human UCK2 (Fig. 8b), although the $\mathrm{pH}$ dependence of human UCK2 activity has not been reported. At the transition state, desolvation of the active site by domain closure might enhance the $\mathrm{p} K_{\mathrm{a}}$ shift of Asp40. Further experiments, including structural analysis using another ATP analogue, are required to fully elucidate the mechanism of UCK.

Acknowledgements This work was partially supported by the Ministry of Education, Science, Sports and Culture, Grant-in-Aid for Challenging Exploratory Research 25650008 (to RM) and the Japan Society for the Promotion of Science for Young Scientists (10J0180400-00, FT).

\section{References}

1. Anderson EP (1973) Nucleoside and nucleotide kinases in The Enzymes (Boyer PD, ed.), 3rd ed., Vol IX, pp. 49-96. Academic Press, New York.

2. O'Donovan GA, Neuhard J (1970) Pyrimidine metabolism in microorganisms. Bacteriol Rev $34: 278-283$

3. Anderson EP, Brockman RW (1964) Feedback inhibition of uridine kinase by cytidine triphosphate and uridine triphosphate. Biochim Biophys Acta 91:380-386

4. Murata D, Endo Y, Obata T, Sakamoto K, Syouji Y, Kadohira M, Matsuda A, Sasaki T (2004) A crucial role of uridine/cytidine kinase 2 in antitumor activity of 3'-ethynyl nucleosides. Drug Metab Dispos 32:1178-1182

5. Orengo A (1969) Regulation of enzymic activity by metabolites. I. Uridine-cytidine kinase of 
Novikoff ascites rat tumor. J Biol Chem 244:2204-2209

6. Shimamoto Y, Koizumi K, Okabe H, Kazuno H, Murakami Y, Nakagawa F, Matsuda A, Sasaki T, Fukushima M (2002) Sensitivity of human cancer cells to the new anticancer ribo-nucleoside TAS-106 is correlated with expression of uridine-cytidine kinase 2. Jpn J Cancer Res 93:825-833

7. Canellakis ES (1957) Pyrimidine metabolism. II. Enzymatic pathways of uracil anabolism. J Biol Chem 227:329-338

8. Sköld O (1960) Uridine kinase from Ehrlich ascites tumor: purification and properties. J Biol Chem 235:3273-3279

9. Liacouras AS, Garvey III TQ, Millar FK, Anderson EP (1975) Uridine cytidine kinase: Kinetic studies and reaction mechanism. Arch Biochem Biophys 168:74-80

10. Yan H, Tsai M (1999) Nucleoside monophosphate kinases: structure, mechanism, and substrate specificity. Adv Enzymol Relat Areas Mol Biol 73:103-134

11. Suzuki NN, Koizumi K, Fukushima M, Matsuda A, Inagaki F (2004) Structural basis for the specificity, catalysis, and regulation of human uridine-cytidine kinase. Structure 12:751-764

12. Tomoike F, Nakagawa N, Kuramitsu S, Masui R (2011) A single amino acid limits the substrate specificity of Thermus thermophilus uridine-cytidine kinase to cytidine. Biochemistry 50:4597-4607

13. Smith AJ, Li Y, Houk KN (2009) Quantum mechanics/molecular mechanics investigation of the mechanism of phosphate transfer in human uridine-cytidine kinase 2. Org Biomol Chem 7:2716-2724

14. Kuramitsu S, Hiromi K, Hayashi H, Morino Y, Kagamiyama H (1990) Pre-steady state kinetics of Escherichia coli aspartate aminotransferase catalized reactions and thermodynamic aspects of its substrate specificity. Biochemistry 29:5469-5476

15. Iino H, Naitow H, Nakamura Y, Nakagawa N, Agari Y, Kanagawa M, Ebihara A, Shinkai A, Sugahara M, Miyano M, Kamiya N, Yokoyama S, Hirotsu K, Kuramitsu S (2008) Crystallization screening test for the whole-cell project on Thermus thermophilus HB8. Acta Cryst F64:487-491

16. Ueno G, Kanda H, Hirose R, Ida K, Kumasaka T, Yamamoto M (2006) RIKEN structural genomics beamlines at the SPring-8; high throughput protein crystallography with automated beamline operation. J Struct Funct Genomics 7:15-22

17. Otwinowski Z, Minor W (1997) Processing of X-ray diffraction data collected in oscillation mode. Methods Enzymol 276:307-326

18. Vagin A, Teplyakov A (1997) MOLREP: an automated program for molecular replacement. J Appl Cryst 30:1022-1025

19. Duncan EM (1992) A visual protein crystallographic software system for X11/Xview. J Mol Graph 10:44-46

20. Brünger AT, Adams PD, Clore GM, DeLano WL, Gros P, Grosse-Kunstleve RW, Jiang J-S, 
Kuszewski J, Nilges M, Pannu NS, Read RJ, Rice LM, Simonson T, Warren GL (1998) Crystallography \& NMR System: A new software suite for macromolecular structure determination. Acta Crystallogr D Biol Cryst 54:905-921

21. Laskowski RA, MacArthur MW, Moss DS, Thornton JM (1993) PROCHECK: a program to check the stereochemical quality of protein structreus. J Appl Crystallogr 26:283-291

22. Kleywegt GJ (2007) Crystallographic refinement of ligand complexes. Acta Crys D Biol Crystallogr 63:94-100

23. Pettersen EF, Goddard TD, Huang CC, Couch GS, Greenblatt DM, Meng EC, Ferrin TE (2004) UCSF Chimera-a visualization system for exploratory research and analysis. J Comput Chem 25:1605-1612

24. Iwai T, Kuramitsu S, Masui R (2004) The Nudix hydrolase Ndx1 from Thermus thermophilus HB8 is a diadenosine hexaphosphate hydrolase with a novel activity. J Biol Chem 279:21732-21739

25. Fersht AR (1999) Structure and Mechanism in Protein Science. Freeman and Company, New York.

26. Bellinzoni M, Haouz A, Graña M, Munier-Lehmann H, Shepard W, Alzari PM (2006) The crystal structure of Mycobacterium tuberculosis adenylate kinase in complex with two molecules of $\mathrm{ADP}$ and $\mathrm{Mg}^{2+}$ supports an associative mechanism for phosphoryl transfer. Protein Sci 15:1489-1493

27. Sekulic N, Shuvalova L, Spangenberg O, Konrad M, Lavie A (2002) Structural characterization of the closed conformation of mouse guanylate kinase. J Biol Chem 277:30236-30243

28. Antosiewicz J, McCammon JA (1996) The determinants of $\mathrm{pK}_{\mathrm{a}} \mathrm{s}$ in proteins. Biochemistry 35:7819-7833

29. Cowan JA (1995) The Biological Chemistry of Magnesium. John Wiley \& Sons, Inc.

30. Picard M, Toyoshima C, Champeil P (2005) The average conformation at micromolar $\left[\mathrm{Ca}^{2+}\right]$ of $\mathrm{Ca}^{2+}$-atpase with bound nucleotide differs from that adopted with the transition state analog ADP.AlFx or with AMPPCP under crystallization conditions at millimolar $\left[\mathrm{Ca}^{2+}\right]$. J Biol Chem 280:18745-18754

31. Taira K, Uebayasi M, Maeda H, Furukawa K (1990) Energetics of RNA cleavage: implications for the mechanism of action of ribozymes. Protein Eng 3:691-702

32. Pörtner HO, Heisler N, Grieshaber MK (1984) Anaerobiosis and acid-base status in marine invertebrates: a theoretical analysis of proton generation by anaerobic metabolism. J Comparative Physiol B 155:1-12

33. Kim YG, Yoo JS, Kim JH, Kim CM, Oh JW (2007) Biochemical characterization of a recombinant Japanese encephalitis virus RNA-dependent RNA polymerase. BMC Mol Biol $8: 59$

34. Karp DA, Stahley MR, Garcia-Moreno B (2010) Conformational consequences of ionization of Lys, Asp, and Glu buried at position 66 in staphylococcal nuclease. Biochemistry 
49:4138-4146 


\section{Figure captions}

Fig. 1 Overall structure of the ternary complex of ttUCK with substrates. Panels $\mathbf{a}$ and $\mathbf{b}$ show a dimer in the asymmetric unit and a monomer, respectively. In panel a, the two subunits are represented in different colors. In panel b, the domains are colored as follows; core, aqua; LID, yellow; NMP-binding, green; and $\beta$ hairpin, salmon.

Fig. 2 Electron density maps of the substrates. Panels $\mathbf{a}$ and $\mathbf{b}$ show the Fo-Fc omit maps of cytidine and AMPPCP, respectively, at $3 \sigma$ above the mean.

Fig. 3 The interactions of ttUCK with cytidine and AMPPCP (the phosphate group) in the ternary complex. a An expanded view of the active site. Carbon atoms of the ligands are colored green. Hydrogen bonds are shown by dashed lines, and their lengths are indicated. The color of the carbon atoms of ttUCK is the same as described for Fig. 1b. The distances (in $\AA$ ) are indicated. b Sequence alignment of UCK homologues. The residues shown in Fig. 3a are indicated by red letters. Conserved residues are indicated by asterisks at the bottom of the alignment. Numbers above the alignment correspond to the amino acid positions in ttUCK. Numbers in parentheses indicate lengths of sequences that were omitted from the figure.

Fig. 4 Conformational change upon substrate binding. Panel $\mathbf{a}$ and $\mathbf{b}$ show the superposition of the ternary complex (green) on the ligand-free (yellow) and on the CMP-bound (blue) forms of ttUCK, respectively.

Fig. 5 The active site of ttUCK. The structures of the ternary and binary complexes are superimposed and colored green and blue, respectively. The distances (in $\AA$ ) between several residues and ligands are described. 
Fig. 6 Effect of mutation at Asp40 on ttUCK activity. a Elution profiles of the reaction products after 1 h. Solid, dash, and dot lines indicate elution profiles of reaction products by WT, D40N and D40A enzyme, respectively. $\mathbf{b}$ Time course of the reaction. A ratio of the peak area of CMP to the total peak area (cytidine and CMP) is plotted against reaction time. The symbols are: squares, WT enzyme; circles, D40N; and crosses, D40A. The reaction conditions for this assay are given under Materials and methods section.

Fig. $7 \mathrm{pH}$ profile of ttUCK activity. The $\mathrm{pH}$ profiles of WT enzyme and H41A are shown as squares and triangles, respectively.

Fig. 8 The positions of Asp40 (a) and Asp62 (b) and surrounding environment in ttUCK and human UCK2. Basic residues, non-charged hydrophilic residues and hydrophobic residues are colored cyan, orange and yellow, respectively. A water molecule in the panel is shown as a red sphere. The distances (in $\AA$ ) are indicated. 
Table 1. Data collection and refinement statistics for ttUCK and its complex with cytidine and AMPPCP

\begin{tabular}{ll} 
Crystal parameters & \\
Space group & $P 2{ }_{1}{ }_{1} 2$ \\
Unit-cell parameters $(\AA)$ & 68.7 \\
a & 126.9 \\
b & 61.7 \\
c & \\
\hline Data processing & $24.00-1.90$ \\
Resolution $(\AA)$ & 284,469 \\
No. of measured reflections & $99.5(100)$ \\
Completeness $(\%)$ & $6.7(6.7)$ \\
Redundancy & $32.27(3.78)$ \\
I/ $\sigma(\mathrm{I})$ & $7.2(52.8)$ \\
$R_{\text {merge }}(\%)^{\dagger}$ & \\
\hline Refinement parameters & $23.48-1.90$ \\
Resolution range $(\mathrm{F}>0)(\AA)$ & 42,184 \\
No. of reflection & 0.208 \\
$R^{\ddagger}$ & 0.243 \\
$R_{\text {free }}$ & 3269 \\
No. of atoms & 219 \\
Protein & 78 \\
Water & 27.0 \\
Ligand & \\
Average B value $\left(\AA^{2}\right)$ & 0.016 \\
RMSD & 1.6 \\
Bond lengths $(\AA)$ & 93.5 \\
Bond angles $\left({ }^{\circ}\right)$ & 6.5 \\
Ramachandran Plot $(\%)$ & 0.0 \\
Most favored & 0.0 \\
Additional allowed & \\
Generously allowed & \\
Disallowed & \\
\hline
\end{tabular}

Values in parentheses are for the outermost shell

${ }^{\dagger} R_{\text {merge }}=\Sigma_{h k l} \Sigma_{i}\left|I_{i}(h k l)-<I(h k l)>\right| / \Sigma_{h k l} \Sigma_{i} I_{i}(h k l)$, where $\langle I(h k l)>$ is the average of individual $I_{i}(h k l)$ measurements

${ }^{\sharp} R=|| \mathrm{F}_{0}|-| \mathrm{F}_{\mathrm{c}}|| / \Sigma\left|\mathrm{F}_{0}\right|$

"root-mean-square deviation 

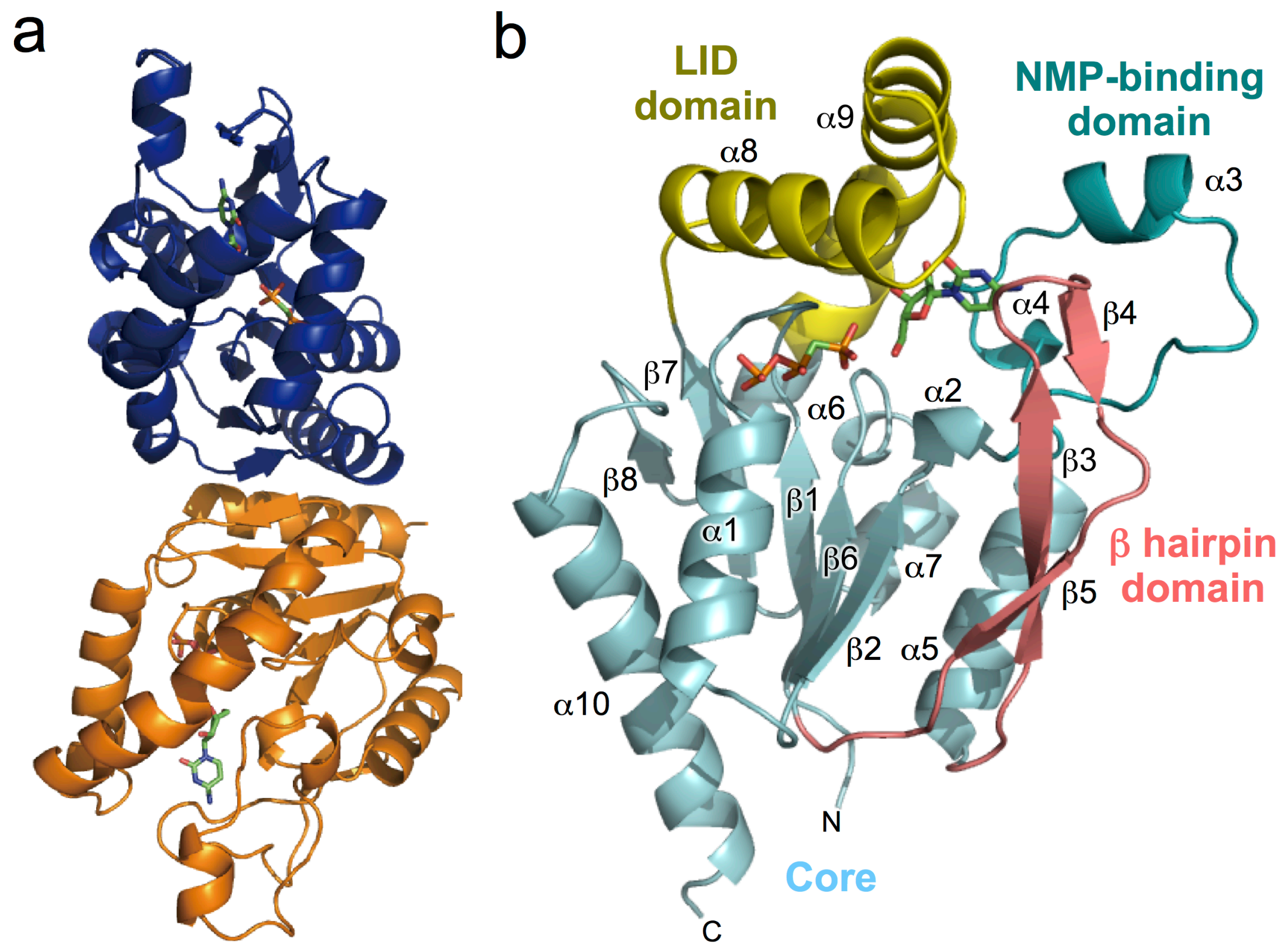

Fig. 1 F. Tomoike 

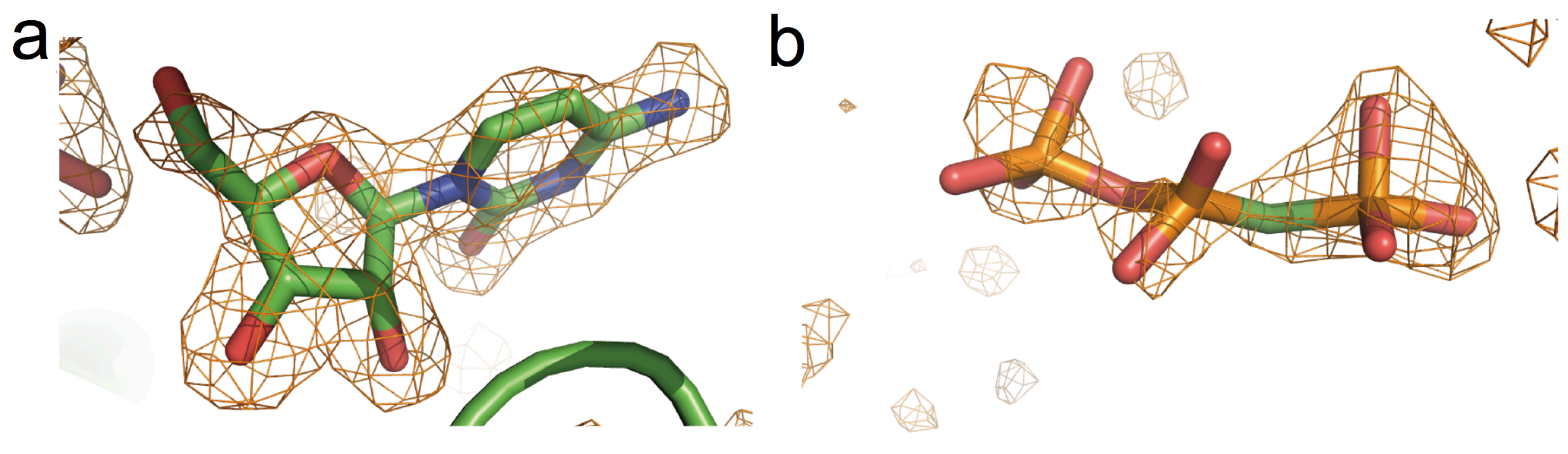

Fig. 2 F. Tomoike 


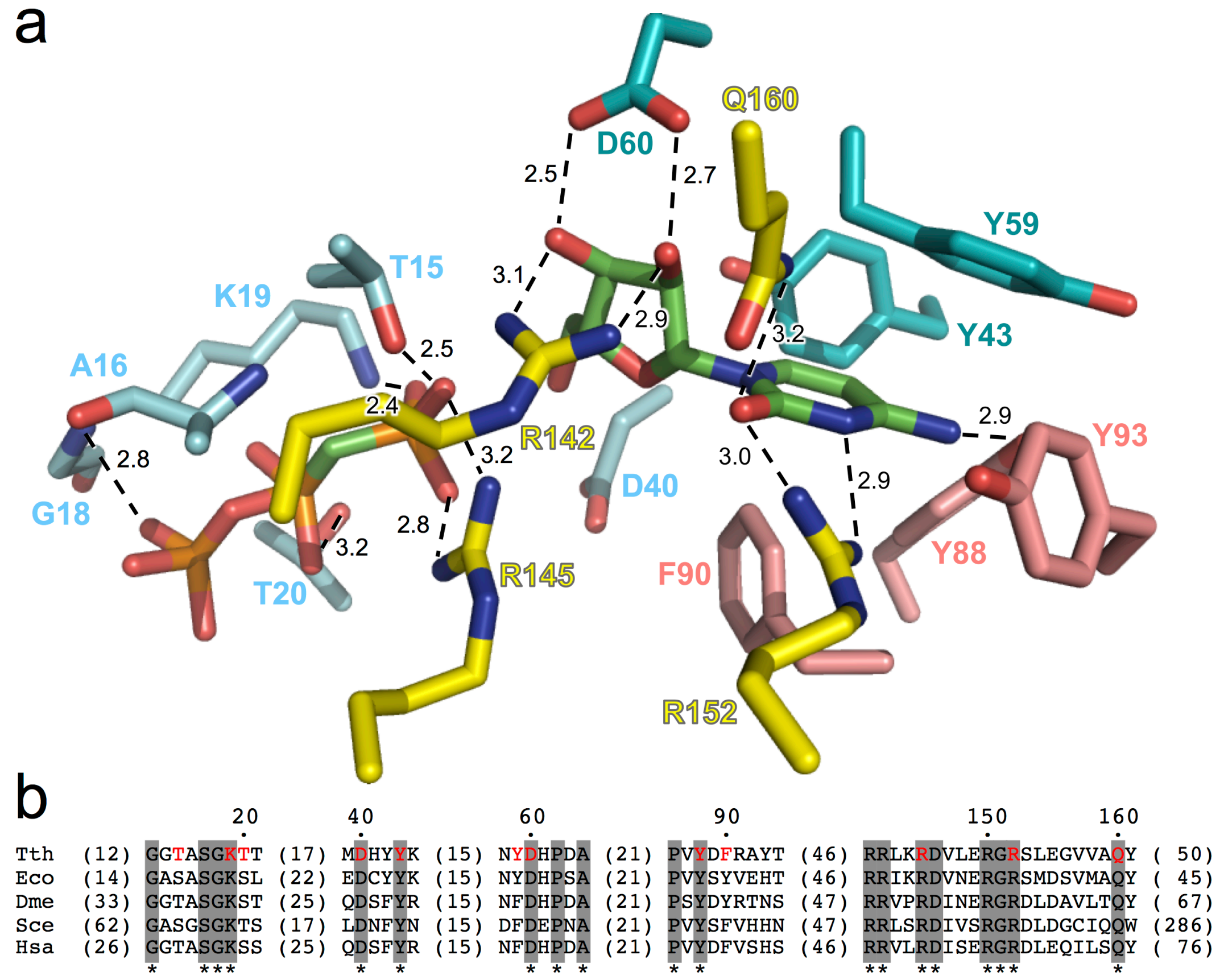


a

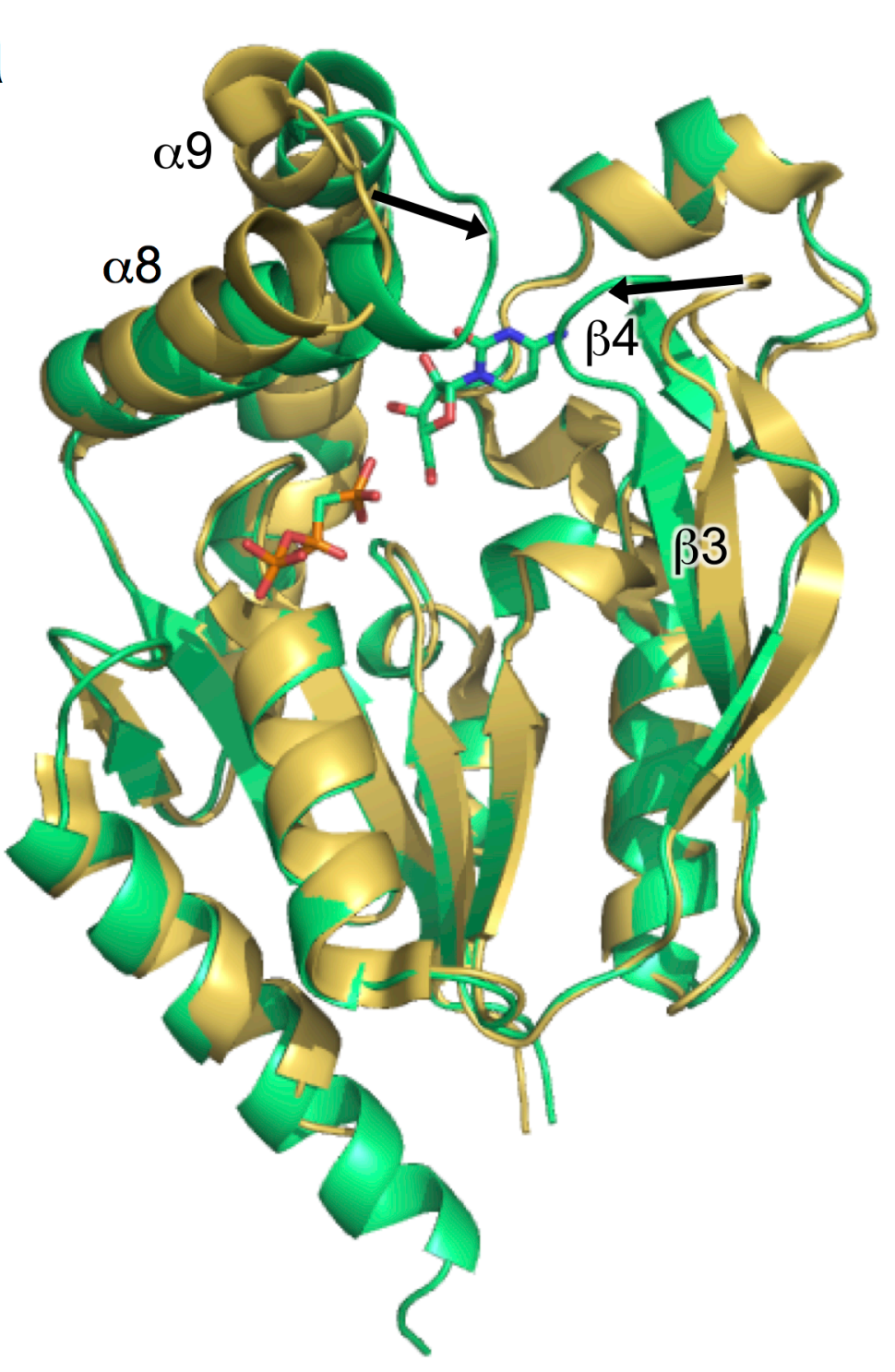

b

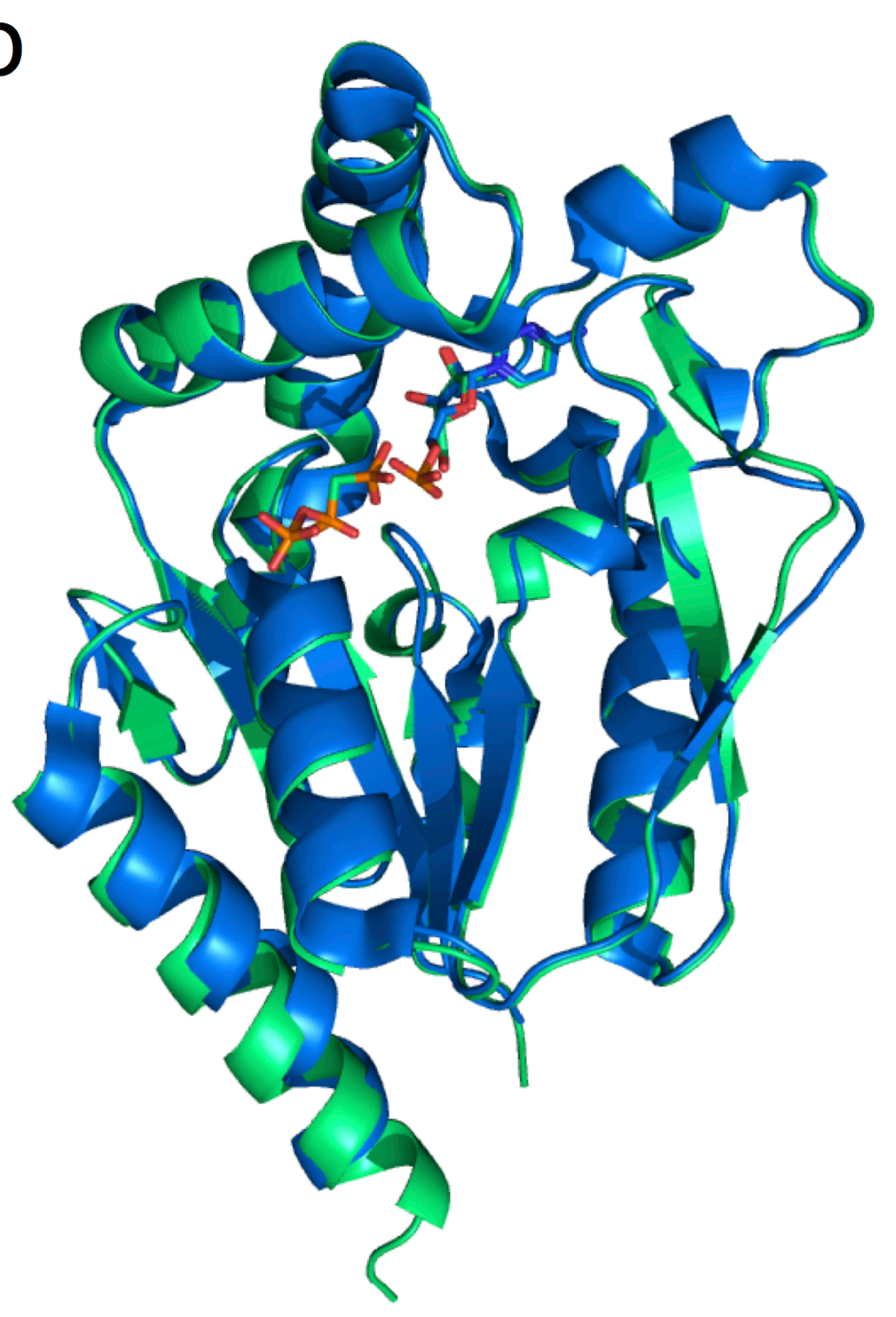

Fig. 4 F. Tomoike 


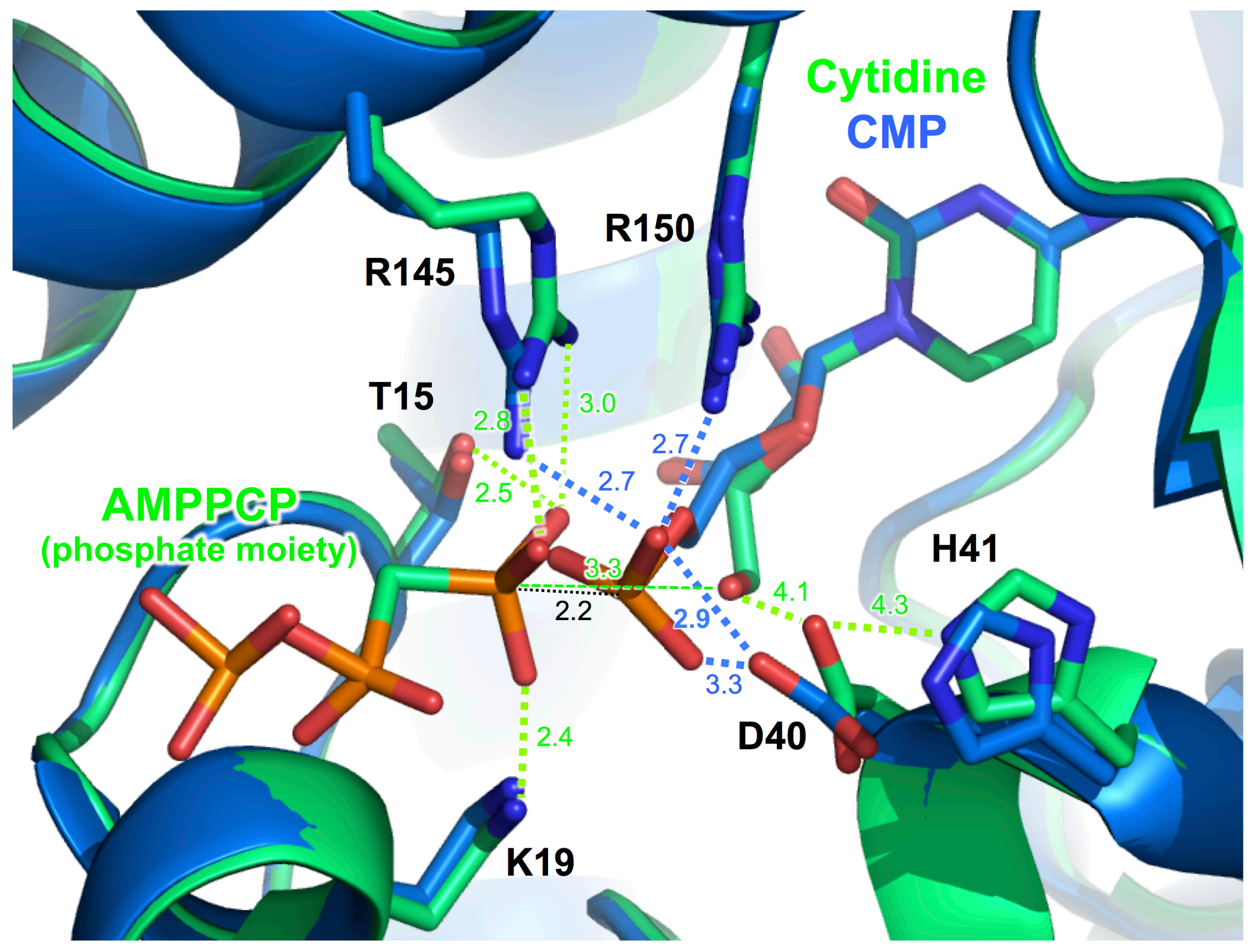

Fig. 5 F. Tomoike 

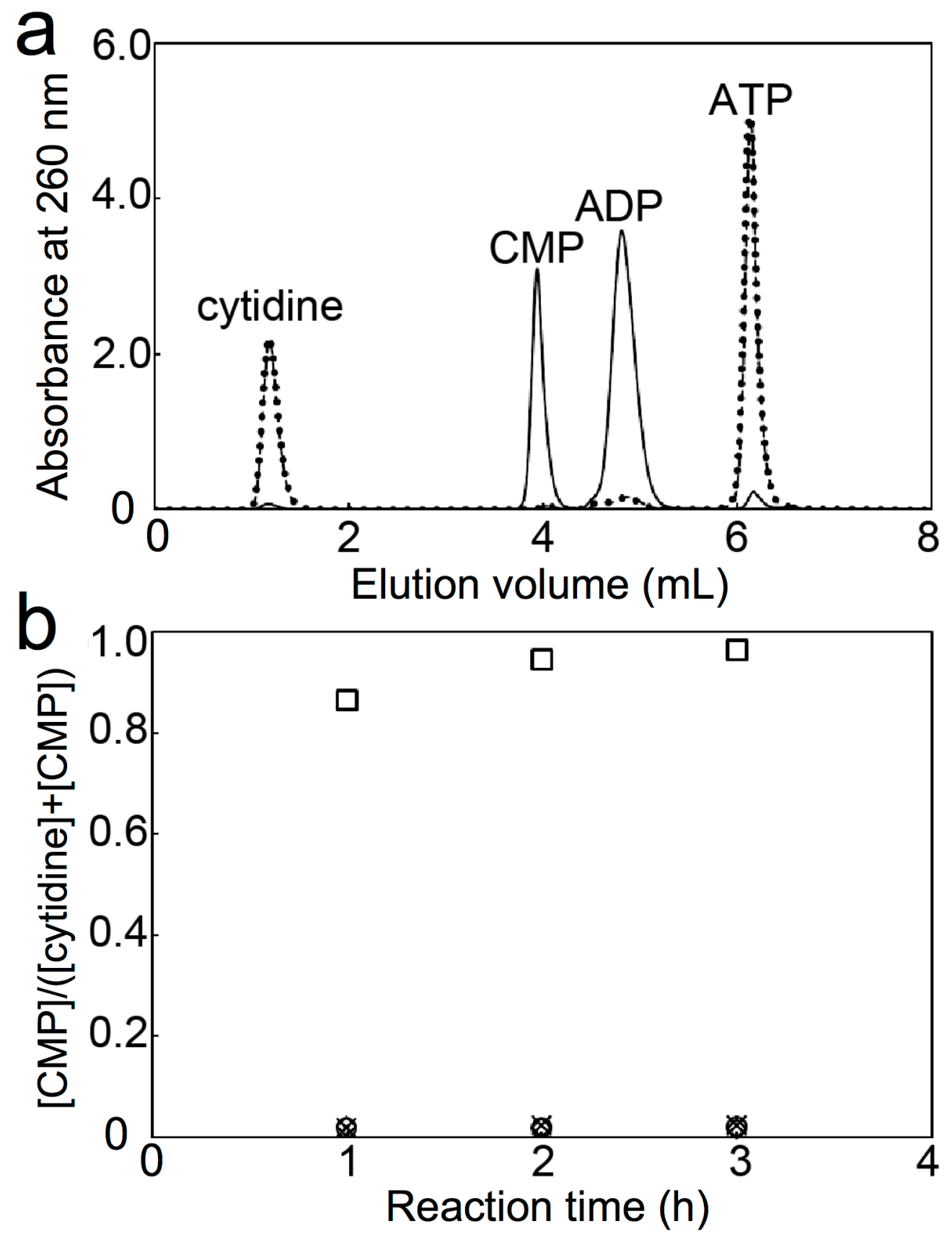

Fig. 6 F. Tomoike 


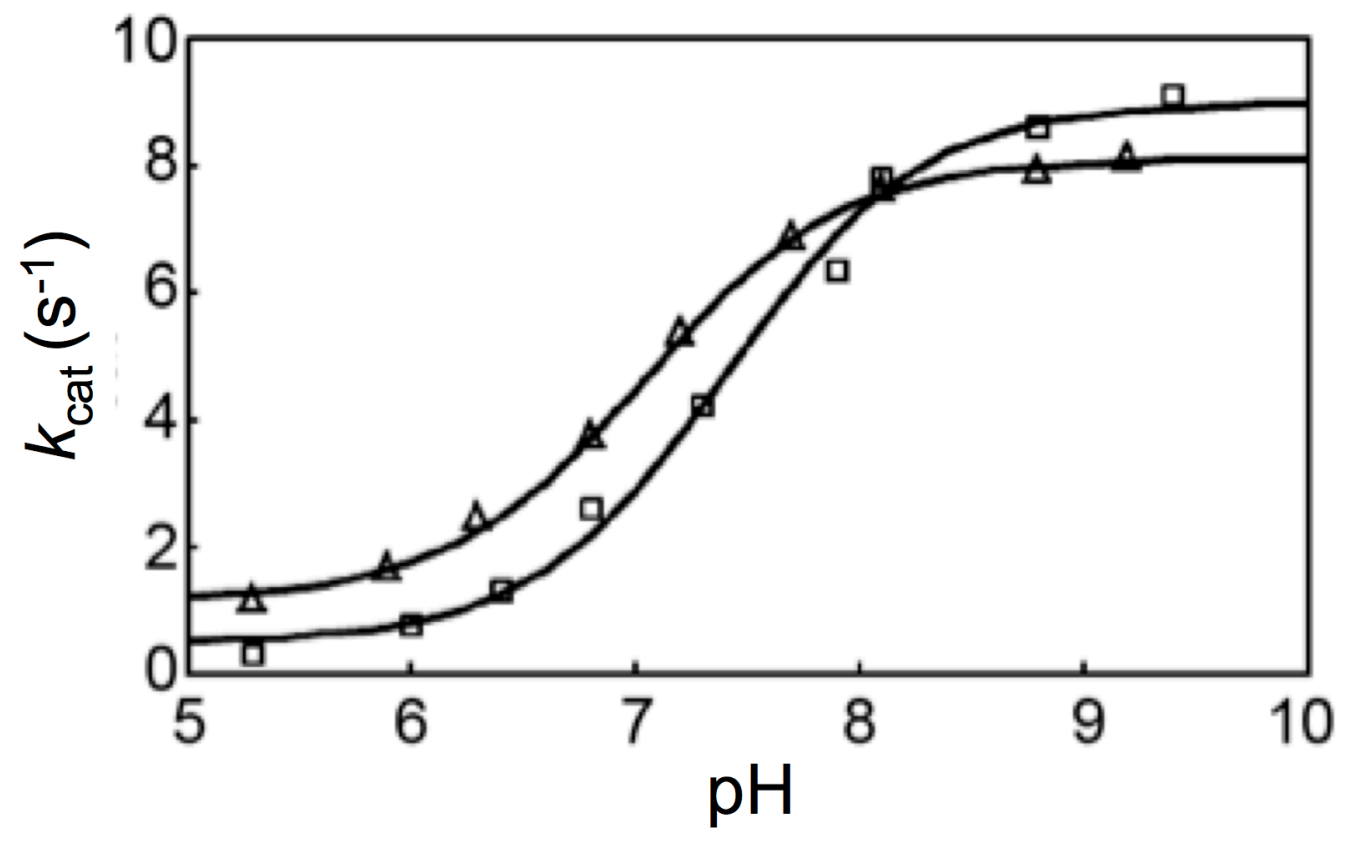

Fig. 7 F. Tomoike 


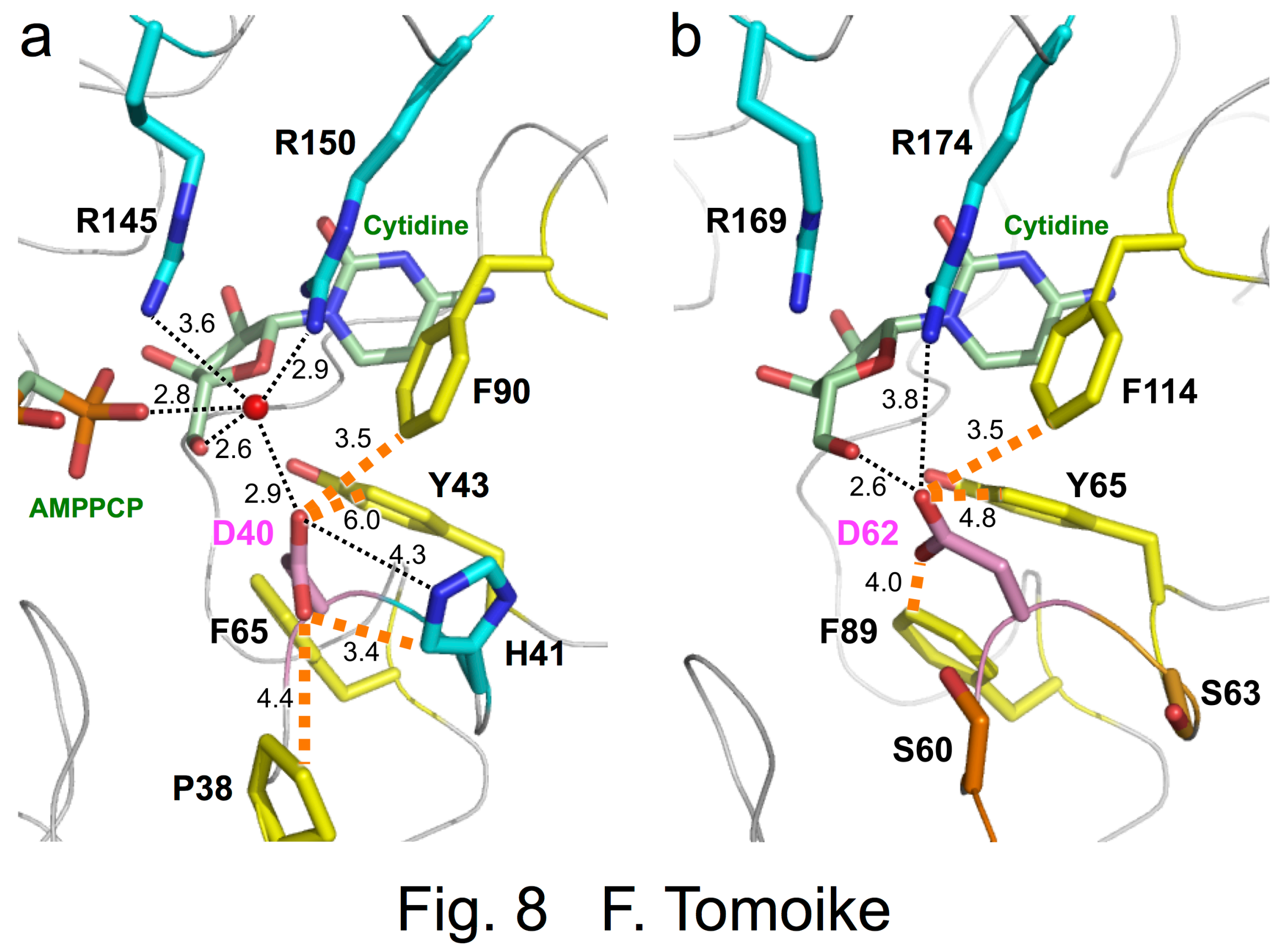

